\subsection{Auszeichnungen}

1960 Goldenes Ehrenzeichen für Verdienste um die Republik Österreich

1963 Goldene Ehrenmünze des Österreichischen Ingenieur- und Architekten-Vereins

1968 Großes Ehrenzeichen für Verdienste um das Bundesland Niederösterreich

1968 Silberne Ehrenmedaille der Handelskammer Niederösterreich

1969 Ehrenkreuz für Wissenschaft und Kunst, I. Klasse

1971 Ehrenring der Marktgemeinde Wieselburg

1973 Goldene Ehrennadel des Bundesverbandes der Dolmetscher und Übersetzer (BDÜ) der Bundesrepublik Deutschland

1973 Diamond Jubilee Medals of the Institute of Linguists (London)

\section{Schrifttum}

[1] Felber, H., Lang, F., Wersig, G.: Terminologie als angewandte Sprachwissenschaft. Gedenkschrift für Univ.-Prof. Dr. Eugen Wüster. München; K. G. Saur. 1979

- S. 15-28: Würdigung der Person und des Wissenschaftlers. - S. 29-57: Bibliographie der Arbeiten Wüsters.

[2] Berlin: VDE Verlag, 1931, $431 \mathrm{~S}$. Erweiterte neue Auflagen 1966 (2. Aufl.) und 1970 (3. Auf.). Die 3. Auflage 1970 erschien bei Bouvier u. Co., Bonn (Beiheft 2 zum „Sprachforum". Zs. für angewandte Sprachwissenschaft).

[3] Wüster, E., Bauer, L. (Hrsg.): Die Allgemeine Terminologielehre. Ein Grenzgebiet zwischen Sprachwissenschaft, Logik, Ontologie, Informatik und den Sachwissenschaften. Linguistics. Den Haag. 1973.

Wüster E.: Einführung in die allgemeine Terminologielehre und terminologische Lexikographie (posthum bearbeitet von Helmuth Felber). Schriftenreihe der Technischen Universität Wien, Band 8 (Teil 1 und 2). Wien: Springer. 1979.

[4] Lang, A.: Bibliografio de libroj kaj artikoloj verkitaj de Eugen Wüster, 1960 und Gedenkschrift (s. Note 1), S. 48-56.

[5] Wüster, E.: Enciklopedia vortaro Esperanta-Germana. Leipzig: Hirt und Sohn. 1923.

[6] Volapük, Ido, Occidental, Latino sine flexione, Novial, Interlingua.

[7] Wüster, E.: Die Vereinfachung der Großschreibung durch Beseitigung willkürlicher Kleinschreibungen. Wien: Die Muttersprache. 1962. Mehrere Nachdrucke bis 1973.

[8] ÖNORM A 2725: Regeln für das Ordnen von Schriftzeichenfolgen (ABC-Regeln). 1982.

[9] Die International Electrotechnical Commission arbeitete seit 1910 an einer zweisprachigen Fachwörterliste. Ab 1923 wurde an einem englisch-französischen Fachwörterbuch der Elektrotechnik gearbeitet. Anfangs in alphabetischer, später in systematischer Anordnung mit alphabetischen Indices.

[10] Wüster, E.: The machine tool: An interlingual dictionary of basic concepts / Dictionnaire multilingue de la Machine-Outil: Notions fondamentales. London: The Technical Press. 1968. - Grundbegriffe der Werkzeugmaschinen. London: The Technical Press. 1968.

[11] Wüster, E.: Bibliography of monolingual scientific and technical glossaries. Volume I: National standards. Paris: Unesco. 1955 .

- Volume II: Miscellaneous sources. Paris: Unesco. 1959.

[12] In den DIN-Mitteilungen des Deutschen Normungsinstituts, Lebende Sprachen (Langenscheidt), Babel.

[13] Lang, F.: Wieselburg, ein Knotenpunkt der internationalen Terminologiearbeit. Sprachforum 3 (1958) Nr. 2.

[14] Zur Zeit in der Simmeringer Hauptstraße 24, 1110 Wien. Die Vorgeschichte beschreibt Wüster in seinen zwei Berichten The Road to Infoterm 1 . Inventory of sources of scientific and technical terminologiy und 2. A plan for establishing an international information center (Clearinghouse) for terminology (Infoterm Series 1). Pullach b. München: Dokumentation Saur KG. 1974.

[15] Wüster, E.: Die sichtbare und die unsichtbare Alphabetisierung der Dezimalklassifikation. Revue de la Documentation 19 (1952) Nr. 2. Wüster, E.: Zur Frage der Alphabetisierung. DK-Mitteilungen 10 (1965), H. 1.

[16] Weltnorm ISO und die deutsche Norm DIN 1463: Richtlinien für die Erstellung und Weiterentwicklung von Thesauri.

[17] Lang, F.: Eröffnungsvortrag in der Gründungssitzung des FNA Bibliothekswesen und Dokumentation im April 1954. Maschinenschrift (Manuskript).

[18] ÖGDB: Dokumentation und Information in Österreich. Dokumentationsführer für Wissenschaft, Technik und Wirtschaft. Wien: Brüder Hollinek. 1970 (1. Aufl. 1964).

[19] Wüster, E.: Die Herstellung der Sägeblätter für Holz. Wien: Springer. 1952.

[20] Richter, L.: Laudatio für Hochschuldozent Dipl--Ing. Dr. techin. Eugen Wüster anläßlich der Verleihung der goldenen Ehrenmünze des Österreichischen Ingenieur- und Architektenvereins (1963). Österr. Ingenieur-Zeitschrift 108 (1963), H. 8. Fritz, A.: Eugen Wüster - 70 Jahre. E und M 85 (1968), H. 10. Lang, F.: Eine Ernte wird eingebracht - Eugen Wüster 75. E und M 90 (1973), H. 9.

Sequenz, H.: Eugen Wüster †. E und M 94 (1977), H. 6.

Felber, H.: In Memoriam Eugen Wüster. Lebende Sprachen 22 (1977), H. 3, S. 141-142.

Lang, F.: Eugen Wüster 1898-1977. ÖGDI-Mitteilungen 7 (1977), Nr. 18.

Lang, F.: Eugen Wüster $\dagger$. - Sein Werk aber lebt weiter. Internationale Klassifikation (1977), H. 1.

\title{
Internationales Symposium: Metrology for Quality Control in Production
}

\section{H. P. Osanna ÖVE ${ }^{1}$}

Metrology for Quality Control in Production war Schwerpunkt-Thema einer internationalen Tagung, an der Experten aus Forschung und Industrie vom 8. bis 10. September 1998

\footnotetext{
Ao. Univ.-Prof. Dipl.-Ing. Dr. P. Herbert Osanna, Konferenz-Vorsitzender, Abteilung Austauschbau und Meßtechnik am Institut für Fertigungstechnik der TU Wien, Karlsplatz 13, A-1040 Wien.
}

im Festsaal der Technischen Universität Wien teilgenommen haben. Mehr als 150 Teilnehmer aus insgesamt 26 Ländern, nämlich

$\begin{array}{ll}\text { Albanien, } & \text { Litauen, } \\ \text { Ägypten, } & \text { Niederlande, } \\ \text { Brasilien, } & \text { Österreich, }\end{array}$


Bulgarien,

Dänamark,

China,

Deutschland,

Finnland,

Großbritannien,

Iran,

Italien,

Japan,

Kroatien,
Polen,

Rumänien,

Rußland,

Schweiz,

Slowakei,

Slovenien,

Tschechische Republik,

Türkei,

Ungarn,

USA

haben diese Veranstaltung besucht. An jedem Tag wurden etwa 30 Vorträge abgehalten. Für Diskussionen stand jeweils ausreichend Zeit beim mittäglichen Arbeits-Imbiß und beim Heurigenabend sowie beim Empfang im Wr. Rathaus zur Verfügung.

Metrology for Quality Control in Production: Die Meßtechnik ist eine „Enabling Science“ - eine Wissenschaft, welche die Basis für präzise Fertigung und weitere Entwicklungen bildet.

Viele Milliarden Dollar werden jährlich in die Entwicklung

der Meßtechnik investiert. Die Herstellung von HiTech-Geräten (z. B. Mobiltelefone, Air-Bags oder Videorecorder) ist ohne die Anwendung modernster und genauester Meßmethoden nicht denkbar.

Die Entwicklung von Qualitätsmanagementsystemen erfordert in hohem Maße die Einbindung der Meßtechnik in die Fertigung. Die Anwendung der Meßtechnik entwickelt sich hierbei von Kontrollmessungen am Ende des Fertigungsprozesses weg in Richtung prozeßbegleitender und prozeßintegrierter Meßtechnik. So kann man etwa seit 1970 die vermehrte Einbeziehung von computerunterstützten Meßtechnologien (z. B. Koordinatenmeßtechnik) beobachten. Ein Ende der Entwicklung der MeBtechnik - sei es im Hinblick auf die Genauigkeit oder im Hinblick auf ihre Bedeutung ist nicht absehbar.

In der April-Ausgabe 1999 der e \& i wird noch genauer über diese Veranstaltung berichtet werden; insbesondere ist vorgesehen, ausgewählte Beiträge zu einigen speziellen Themen in vollständiger Form zum Abdruck zu bringen.

\section{Zentralstatistik elektrischer Unfälle für das Jahr 1997}

Mitgeteilt vom Bundesministerium für wirtschaftliche Angelegenheiten (in gekürzter Form wiedergegeben)

\section{Unfälle durch Elektrizität}

1.1 Gesamtanzahl der erfaßten Unfälle durch elektrischen Strom

1997

$$
\begin{aligned}
& \text { Anzahl der } \\
& \text { Unfälle } \\
& \text { gesamt }
\end{aligned}
$$

172
Mit tödlichem

Ausgang

14

\subsection{Art der Unfälle}

Arbeitsunfall .................. 150

Wegunfall $\ldots \ldots \ldots \ldots \ldots \ldots \ldots \ldots \ldots \ldots \ldots$.

Privatunfall ... . . . . . . . . . . . . . . . . . . 19

Absichtlich herbeigeführter Unfall ..... 1

Unbekannt $\ldots \ldots \ldots \ldots \ldots \ldots \ldots \ldots \ldots .2$

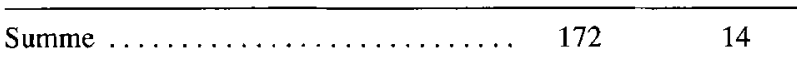

\begin{tabular}{|c|c|c|}
\hline $\begin{array}{l}\text { Männlich } \ldots \ldots \ldots \ldots \ldots \ldots \ldots \ldots \ldots \\
\text { Weiblich } \ldots \ldots \ldots \ldots \ldots\end{array}$ & $\begin{array}{r}156 \\
16\end{array}$ & 14 \\
\hline Summe . . & 172 & 14 \\
\hline
\end{tabular}

\subsection{Geschlecht der Verletzten}

\subsection{Berufsarten}

Elektroingenieure und Betriebsleiter . . . . 3

Elektromeister $\ldots \ldots \ldots \ldots \ldots \ldots \ldots \ldots . \ldots \ldots, 12$

Obermonteure .................. 2

Monteure (auch Elektroinstallateure,

Elektriker) . . . . . . . . . . . .

Hilfsmonteure (Angelernte)

Lehrlinge

-

1

14

(

